ARTIGO ORIGINAL

\title{
Diversidade de basidiomicetos macroscópicos em áreas de reflorestamento de Eucalyptus spp.
}

\author{
Diversity of macroscopic basidiomycetes in reforestation areas of \\ Eucalyptus spp.
}

Beatriz Lourenço Manzato ${ }^{1}$, Caroline Lourenço Manzato ${ }^{1}$ (D), Paula Leite dos Santos ${ }^{1}$ (D), José Raimundo de Souza Passos ${ }^{1}$ [C], Tadeu Antônio Fernandes da Silva Júnior ${ }^{1}$ (1)

'Universidade Estadual Paulista “Julio de Mesquita Filho" - UNESP, Botucatu, SP, Brasil

Como citar: Manzato, B. L., Manzato, C. L., Santos, P. L., Passos, J. R. S., \& Silva Júnior, T. A. F. (2020). Diversidade de basidiomicetos macroscópicos em áreas de reflorestamento de Eucalyptus spp. Scientia Forestalis, 48(128), e3305. https://doi.org/10.18671/scifor.v48n128.08

\begin{abstract}
Resumo
O eucalipto (Eucalyptus spp.) é o gênero florestal mais utilizado para fins comerciais em nível mundial. Uma das maiores preocupações dos silvicultores são os resíduos deixados na área após o corte do eucalipto. Na tentativa de solucionar o problema, produtores optam pelo método de rebaixamento ou remoção de tocos. Entretanto, a destoca mecanizada causa inúmeros impactos negativos à sustentabilidade florestal, entre eles, compactação do solo; perturbação da microbiota; remoção de matéria orgânica; impactos no armazenamento do carbono; emissão de gases de efeito estufa; aumento na erosão; alteração da ciclagem de nutrientes e redução da biodiversidade. O emprego de fungos degradadores de madeira é uma alternativa para a remoção dos tocos, sem impactar negativamente o ambiente, porém é pouco estudado e empregado em áreas de reflorestamento de eucalipto. Este trabalho se destaca por realizar o levantamento da diversidade de fungos basidiomicetos macroscópicos em tocos e na serrapilheira, em áreas de reflorestamento de eucalipto de diferentes idades (tocos pós-colheita e com um e dois anos após a colheita) no interior do Estado de São Paulo para utilização na destoca biológica. Os fungos foram coletados entre setembro de 2016 a agosto de 2017, totalizando 16 coletas, onde cada fungo foi fotografado para identificação morfológica presuntiva. Os fungos de maior ocorrência nos tocos com dois anos após a colheita foram os pertencentes aos gêneros Coprinus e Ganoderma. Na área com os tocos com um ano após a colheita houve predomínio dos gêneros Coprinuse Galerina. Não foram encontrados fungos na área com tocos pós colheita. Houve maior diversidade de fungos basidiomicetos macroscópicos na área de reflorestamento de eucalipto com tocos com dois anos após a colheita.
\end{abstract}

Palavras-chave: Destoca biológica; Floresta plantada; Eucaliptocultura; Resíduos florestais; Bioprospecção.

\begin{abstract}
Eucalyptus (Eucalyptus spp.) Is the most widely used genus of forest trees for commercial purposes worldwide. One of the biggest concerns for foresters is the waste left in the area after cutting the eucalyptus. In an attempt to solve the problem, producers opt for the method of lowering or removing stumps. However, mechanized logging causes numerous negative impacts on forest sustainability, including soil compaction; microbiota disturbance; removal of organic matter; impacts on carbon storage; greenhouse gas emissions; increased erosion; alteration of nutrient
\end{abstract}

Fonte de financiamento: O presente trabalho foi realizado com apoio da Coordenação de Aperfeiçoamento de Pessoal de Nível Superior - Brasil (CAPES) Código de Financiamento 001.

Conflito de interesse: Nada a declarar.

Autor correspondente: beatrizmanzato@hotmail.com

Recebido: 5 março 2019.

Aceito: 22 outubro 2019.

Editor: Paulo Henrique Müller Silva.

(c) Este é um artigo publicado em acesso aberto (Open Access) sob a licença Creative Commons Attribution, que permite uso, distribuição e reprodução em qualquer meio, sem restrições desde que o trabalho original seja corretamente citado. 
cycling and reduction of biodiversity. The use of wood degrading fungi is an alternative for the removal of stumps, without negatively impacting the environment, but it is little studied and used in eucalyptus reforestation areas. This work stands out for surveying the diversity of macroscopic basidiomycete fungi in stumps and litter in eucalyptus reforestation areas of different ages (postharvest stumps and one and two years after harvest) in the interior of São Paulo State for use in biological removal. The fungi were collected from September 2016 to August 2017, totaling 16 collections, where each fungus was photographed for presumptive morphological identification. The most common fungi in the stumps two years after harvesting were those belonging to the genera Coprinus and Ganoderma. In the area with stumps one year after harvesting, there was a predominance of the genera Coprinus and Galerina. No fungi were found in the area with postharvest stumps. There was a greater diversity of macroscopic basidiomycete fungi in the area of eucalyptus reforestation with stumps two years after harvest.

Keywords: Biological stump removal; Planted forest; Eucalyptus forest; Forest residues; Bio-prospecting.

\section{INTRODUÇÃO}

O eucalipto é um dos gêneros florestais empregados em diversos setores, além de possuir ampla diversidade de espécies (Wilcken et al., 2008). No Brasil, a maior parte das florestas plantadas é composta por eucalipto (Eucalyptus spp.) (Empresa Brasileira de Pesquisa Agropecuária, 2014), sendo que em 2016, a área total plantada foi de 5,7 milhões de hectares, destacando-se os estados de Minhas Gerais (24\%), São Paulo (17\%) e Mato Grosso do Sul (15\%) (Indústria Brasileira de Árvores, 2017).

O corte do eucalipto ocorre em torno dos sete anos de idade (Empresa Brasileira de Pesquisa Agropecuária, 2014; Mora \& Garcia, 2000), e geralmente resíduos da própria cultura permanecem no local após a colheita, principalmente os tocos (ou cepas), sendo estes, um dos principais problemas enfrentados pelos silvicultores. O toco ou cepa é um resíduo do tronco que fica ligeiramente acima do solo e abaixo da terra com suas raízes. Quando uma floresta de eucalipto possui um manejo adequado e a colheita é realizada de acordo com as especificações de altura de toco, estes permanecem na área com elevações entre 5 e $15 \mathrm{~cm}$ acima do solo (Foelkel, 2014).

Um dos métodos adotados na tentativa de solucionar o problema, é a destoca mecanizada por meio do rebaixamento ou extração dos tocos e raízes (Foelkel, 2014; Casselli et al., 2018). Porém, essas práticas não são economicamente viáveis, pois demandam alto investimento e tempo para o processo, e causam inúmeros impactos negativos em relação à sustentabilidade florestal (Casselli et al., 2018).

Os fungos, principalmente os basidiomicetos exercem uma importante atividade ecológica na decomposição de madeira e outros materiais derivados de fontes naturais (Madigan et al., 2016). Nas florestas, os fungos basidiomicetos são importantes no processo de destoca de eucalipto (Magalhães \& Milagres, 2008).

O objetivo deste estudo foi identificar a diversidade de fungos basidiomicetos macroscópicos em tocos e na serrapilheira de Eucalyptus spp. de diferentes idades em áreas de reflorestamento.

\section{MATERIAL E MÉTODOS}

O estudo foi desenvolvido em áreas de reflorestamento de Eucalyptus spp.de uma empresa de celulose, localizada entre os municípios de Lençóis Paulista $\left(22^{\circ} 35^{\prime} 55^{\prime \prime}\right.$ de latitude Sul e $48^{\circ} 48^{\prime} 01^{\prime \prime}$ de longitude Oeste e altitude de 560 metros) e Borebi ( $22^{\circ} 33^{\prime} 52^{\prime \prime}$ de latitude Sul e $48^{\circ} 58^{\prime} 5^{\prime \prime}$ de longitude Oeste e altitude de 590 metros), ambos localizados na região Centro-Oeste do Estado de São Paulo (Figura 1).

Os fungos basidiomicetos foram obtidos por meio de coletas em talhões de Eucalyptus spp. com tocos pós-colheita (área A) e com um e dois anos após a colheita (áreas B e C, respectivamente) (Tabela 1). 


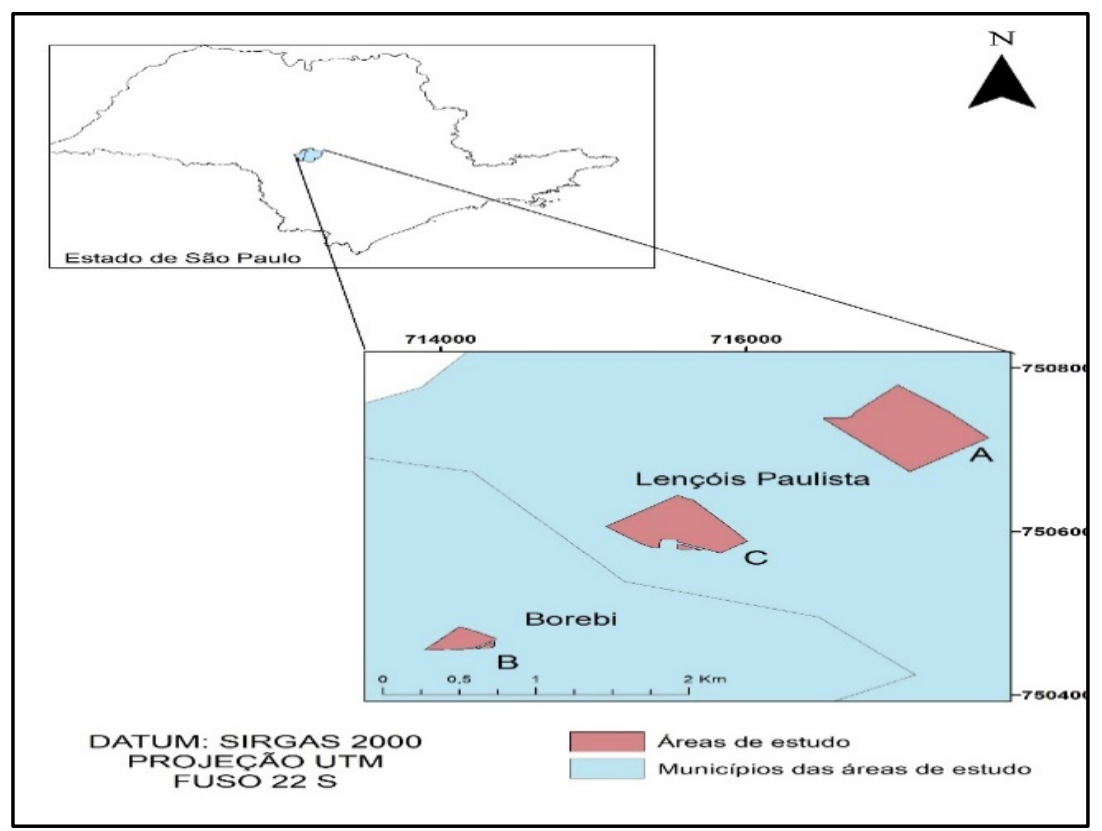

Figura 1. Mapa de localização das áreas de estudo. Sendo (A) área com talhões de eucalipto com tocos pós-colheita; (B) área com talhões de eucalipto com tocos com um ano após a colheita; (C) área com talhões de eucalipto com tocos com dois anos após a colheita.

Tabela 1. Espécies florestais, espaçamento e localização dos talhões de Eucalyptus spp. referentes às áreas de coleta dos fungos basidiomicetos macroscópicos em tocos e na serrapilheira em áreas de reflorestamento.

\begin{tabular}{cccc}
\hline Área de Coleta & Espécie Florestal & Espaçamento (metros) & Município \\
\hline A & Eucalyptus urograndis & $3,8 \times 2,1$ & Lençóis Paulista, SP \\
B & Eucalyptus urophylla & $3,8 \times 2,1$ & Borebi, SP \\
C & Eucalyptus urophylla & $3,8 \times 2,1$ & Lençóis Paulista, SP \\
\hline
\end{tabular}

Nota: Área de coleta A (tocos pós-colheita), área B (tocos com dois anos após a colheita) e área C (tocos com dois anos após a colheita).

Cada área foi dividida em três subáreas, e em cada subárea foram vistoriados 20 tocos nas linhas $x 5$ tocos nas entrelinhas, com uma dimensão de $798 \mathrm{~m}^{2}$, espaçadas 200 metros entre si. Nessas mesmas áreas também foi vistoriada a serapilheira.

Os fungos foram coletados entre setembro de 2016 a agosto de 2017. Cada fungo coletado foi fotografado no local de ocorrência, acondicionado em sacos de papel de $500 \mathrm{~g}$, e mantidos em caixa de isopor para transporte até o Laboratório de Ciência e Tecnologia Ambiental da Universidade do Sagrado Coração. Foram mensurados em cada coleta os parâmetros temperatura $\left({ }^{\circ} \mathrm{C}\right)$, umidade relativa do ar (\%) e luminosidade (Ix).

\section{Metodologia estatística}

\section{Análise descritiva}

Foram realizadas análises de frequência de identificação (gêneros e espécies) de fungos basidiomicetos macroscópicos com a quantidade de indivíduos. Foram obtidas as correlações de Spearman entre as quantidades acumuladas de espécies ou gêneros de basidiomicetos macroscópicos com as variáveis climáticas: temperatura do ar $\left({ }^{\circ} \mathrm{C}\right)$, umidade relativa do ar $(\%)$ e luminosidade (Ix). 
Modelos de regressão da densidade acumulada de fungos basidiomicetos macroscópicos no tempo

Para a área $B$ (tocos com um ano após a colheita), foi ajustado um modelo de regressão linear simples para a densidade acumulada de indivíduos no tempo (dias após a colheita do eucalipto), expresso pela Equação 1:

$$
y_{i}=\alpha+\beta t+u_{i}
$$

em que,

$y_{i}$ é a densidade acumulada de indivíduos na i-ésima observação; $t$ é o tempo em dias;

$\alpha$ e $\beta$ são parâmetros a serem estimados. O parâmetro $\beta$ representa a velocidade de crescimento da densidade acumulada; $u_{i}$ é o componente aleatório com distribuição normal e variância constante.

Para a área C (tocos com dois anos após a colheita), foi ajustado um modelo de regressão não linear logístico (Seber \& Wild, 2003) para a densidade acumulada de indivíduos no tempo, expresso pela Equação 2:

$$
y_{i}=\frac{\alpha}{1+\exp (\beta-\gamma t)}+u_{i}
$$

em que,

$y_{i}$ é a densidade acumulada de indivíduos na i-ésima observação; $e$ é a base dos logaritmos Neperianos; $t$ é o tempo em dias;

$\alpha, \beta$ e $\gamma$ são parâmetros a serem estimados. O parâmetro $\alpha$ representa a assíntota da curva;

$u_{i}$ é o componente aleatório com distribuição normal e variância constante.

Para ajuste do modelo, foi utilizado o procedimento nlin do programa estatístico SAS Free Statistical Statistical Software, SAS University Edition.

\section{RESULTADOS E DISCUSSÃO}

Em todas as coletas realizadas e em todas as áreas vistoriadas foram obtidos 70 basidiomicetos macroscópicos, sendo $72,9 \%$ provenientes dos tocos e $27,1 \%$ da serrapilheira.

$\mathrm{Na}$ área com tocos pós-colheita não foram encontrados basidiomicetos tanto nos tocos, quanto na serrapilheira. A ausência dos basidiomicetos pode ter sido em consequência da maior incidência de luz solar na área em relação às outras áreas, que possuíam maior sombreamento devido à idade do povoamento.

Os fungos que ocorreram nos tocos foram os pertencentes aos gêneros Coprinus, Coriolopsis, Fuscoporia, Galerina, Ganoderma e Pycnoporus. O maior número de indivíduos de basidiomicetos macroscópicos coletados nos tocos de eucalipto em áreas de reflorestamento foram nas coletas 1 (inverno) e 3 (primavera) (Tabela 2).

Sobre a ocorrência dos fungos basidiomicetos encontrados nos tocos, na área com tocos com um ano após a colheita foram coletados 10 indivíduos, sendo os gêneros Coprinus e Galerina de maior ocorrência. Na área com tocos com dois anos após a colheita foram coletados 41 indivíduos, sendo o gênero Ganoderma de maior ocorrência (Tabela 2, supracitada). O gênero Ganoderma pertence à família Ganodermataceae, e os fungos desta família são causadores de podridão branca na madeira ou estão associados a raízes de árvores vivas ou mortas (Furtado, 1981; Ryvarden, 2004), ou seja, degradam todos os componentes primários da madeira, inclusive a lignina (Blanchette, 2000). Portanto, o fungo Ganoderma spp. possui potencial para ser empregado na destoca microbiológica, devido ao seu potencial em degradar os principais constituintes da madeira. 
Tabela 2. Frequência de ocorrência dos gêneros de fungos basidiomicetos macroscópicos coletados em tocos de Eucalyptus spp. provenientes de áreas de reflorestamento em diferentes estações do ano.

\begin{tabular}{ccccccccc}
\hline \multirow{2}{*}{ Gêneros } & \multicolumn{3}{c}{ Áreas } & & \multicolumn{5}{c}{ Estações do ano } \\
\cline { 2 - 4 } \cline { 6 - 8 } & B & C & & Primavera & Verão & Outono & Inverno \\
\hline Coprinus spp. & 5 & 13 & & 8 & 0 & 0 & 10 \\
Coriolopsis spp. & 1 & 1 & & 0 & 1 & 1 & 0 \\
Fuscoporia sp. & 0 & 1 & & 1 & 0 & 0 & 0 \\
Galerina spp. & 4 & 2 & & 6 & 0 & 0 & 0 \\
Ganoderma spp. & 0 & 15 & 1 & 1 & 0 & 13 \\
Pycnoporus spp. & 0 & 9 & 4 & 0 & 0 & 5 \\
Total & 10 & 41 & 20 & 2 & 1 & 28
\end{tabular}

Nota: Área B refere-se à área com tocos com um ano após a colheita e área C à área com tocos com dois anos após a colheita.

Os fungos dos gêneros Coprinus, Coriolopsis e Galerina ocorreram tanto nos tocos com um ano após a colheita (área B), quanto nos tocos com dois anos após a colheita (área C). Os fungos pertencentes aos gêneros Fuscoporia, Ganoderma e Pycnoporus ocorreram apenas nos tocos com dois anos após a colheita (área C) (Tabela 2, supracitada).

$\mathrm{Na}$ área $\mathrm{B}$ o maior número de fungos coletados foi na primavera com 8 indivíduos, e na área C, a maior ocorrência de fungos basidiomicetos nos tocos foi no inverno (Tabela 2, supracitada). A diferença da ocorrência dos fungos entre as áreas pode ser explicada pelas condições de umidade e temperatura serem distintas em cada época do ano (Costa et al., 2011).

Ao observar no tempo (dias após a colheita do eucalipto) a ocorrência dos basidiomicetos macroscópicos nos tocos, ou seja, quanto tempo (em dias) levou até a primeira ocorrência de basidiomicetos macroscópicos nos tocos de eucalipto, pôde-se identificar que a primeira ocorrência de fungos basidiomicetos macroscópicos nos tocos de eucalipto foi aos 365 dias após a colheita, e a maior ocorrência aos 736 dias após a colheita de eucalipto.

Sobre a ocorrência dos fungos basidiomicetos encontrados na serrapilheira, a área $B$ apresentou a ocorrência de apenas 7 indivíduos, que foram observados em apenas uma das coletas realizadas (no outono) com predomínio do gênero Gloeoporus. Esse gênero também foi relatado em troncos caídos em fragmentos florestais do noroeste paulista (Abrahão et al., 2012), e do parque estadual da Cantareira em São Paulo (Motato-Vásquez et al., 2014). Na Mata Atlântica do nordeste brasileiro, foram encontrados dois exemplares desse gênero em angiospermas em decomposição (Gibertoni et al., 2004). Pelo exposto, o fungo Gloeoporus spp. ocorre naturalmente na madeira de diferentes espécies florestais, podendo assim ser empregado na destoca biológica.

Os gêneros Scleroderma, Phellinus, Trametes, Microporellus, Polyporus, Lentinus e Pycnoporus também foram relatados em povoamentos de eucalipto na Bahia por Costa et al. (2011), e os fungos Pycnoporus sanguineus e Lentinus bertieri em campos de reflorestamento de eucalipto no estado de São Paulo por Negrão et al. (2014). E os fungos dos gêneros Peniophora, Pestalotiopsis, Ganoderma e da espécie Pycnoporus sanguineus foram identificados por Alonso et al. (2007) em plantios comerciais de eucalipto localizados em diversos estados do Brasil. Os fungos pertencentes aos gêneros Scleroderma, Phellinus, Trametes, Microporellus, Polyporus, Lentinus, Ganoderma, Peniophora, Pestalotiopsis e Pycnoporus ocorrem naturalmente em plantios de Eucalyptus spp., portanto, podem ser considerados fungos com potencial para utilização na destoca biológica desta espécie florestal por ocorrerem naturalmente nesses locais e degradar esse substrato (Tabela 3).

Na serrapilheira da área 3 foram encontrados 12 basidiomicetos macroscópicos, sendo os fungos Gloeoporus spp., Lentinus spp. e Polyporus spp. de maior ocorrência. O fungo pertencente ao gênero Polyporus foi o único basidiomiceto macroscópico a ocorrer tanto na serrapilheira da área com tocos com um ano após a colheita, quanto da área com tocos com dois anos após a colheita (Tabela 3). 
Tabela 3. Frequência dos gêneros de fungos basidiomicetos macroscópicos coletados da serrapilheira de áreas de reflorestamento de Eucalyptus spp. com tocos pós-colheita e com um e dois anos após a colheita, de diferentes estações do ano.

\begin{tabular}{|c|c|c|c|c|c|c|}
\hline \multirow{2}{*}{ Gêneros } & \multicolumn{2}{|c|}{ Áreas } & \multicolumn{4}{|c|}{ Estações do ano } \\
\hline & B & C & Primavera & Verão & Outono & Inverno \\
\hline Coriolopsis spp. & 0 & 1 & 0 & 1 & 0 & 0 \\
\hline Galerina spp. & 0 & 1 & 0 & 1 & 0 & 0 \\
\hline Ganoderma spp. & 0 & 1 & 0 & 1 & 0 & 0 \\
\hline Gloeoporus spp. & 3 & 0 & 0 & 0 & 3 & 0 \\
\hline Lentinus spp. & 0 & 4 & 0 & 1 & 0 & 3 \\
\hline Microporellus spp. & 2 & 0 & 0 & 0 & 2 & 0 \\
\hline Phellinus spp. & 1 & 0 & 0 & 0 & 1 & 0 \\
\hline Polyporus spp. & 1 & 2 & 0 & 2 & 1 & 0 \\
\hline Scleroderma spp. & 0 & 2 & 0 & 2 & 0 & 0 \\
\hline Trametes spp. & 0 & 1 & 0 & 1 & 0 & 0 \\
\hline Total & 7 & 12 & 0 & 9 & 7 & 3 \\
\hline
\end{tabular}

Nota: Área B refere-se à área com tocos com um ano após a colheita e área C à área com tocos com dois anos após a colheita.

Na serrapilheira foram encontrados fungos dos gêneros Coriolopsis, Galerina, Ganoderma, Gloeoporus, Lentinus, Microporellus, Phellinus, Polyporus, Scleroderma e Trametes (Tabela 3, supracitada).

Ao analisar no tempo (dias após a colheita) a ocorrência dos basidiomicetos macroscópicos da serrapilheira nas áreas de reflorestamento de eucalipto, ou seja, quanto tempo (em dias) levou até a primeira ocorrência de basidiomicetos macroscópicos na serapilheira, pôde-se notar que a primeira ocorrência de fungos basidiomicetos macroscópicos foi aos 560 dias após a colheita do eucalipto.

Ao comparar a ocorrência de fungos basidiomicetos macroscópicos nos dois substratos (tocos e serrapilheira), os fungos Coprinus spp., Fuscoporia sp. e Pycnoporus spp. foram os que ocorreram somente nos tocos. E os fungos Gloeoporus spp., Lentinus spp., Microporellus sp., Phellinus sp., Polyporus spp., Scleroderma spp. e Trametes sp. ocorreram apenas na serrapilheira.

Os parâmetros climáticos obtidos nas coletas de maior ocorrência de fungos basidiomicetos dos tocos, a área $C$ (primeira coleta) apresentou as seguintes médias: temperatura de $26^{\circ} \mathrm{C}$ e umidade relativa do ar de $33 \%$, e a área B (terceira coleta) temperatura de $26^{\circ} \mathrm{C}$ e umidade relativa do ar de $60 \%$ (Tabela 4).

Os fungos se desenvolvem de forma seletiva na madeira entre a variação e a interação de diversas condições ambientais, entre elas estão temperatura e teor de umidade. A temperatura ideal para o desenvolvimento de fungos xilófagos é de $24^{\circ} \mathrm{C}$ a $32^{\circ} \mathrm{C}$, e os teores de umidade variam de $20 \%$ a $80 \%$ (Moreschi, 2013). Analisando os parâmetros ideais (temperatura e teor de umidade) para o desenvolvimento de fungos degradadores de madeira e os resultados obtidos, pôde-se observar que os valores de temperatura e umidade do ar estavam dentro do esperado para esse grupo de fungos, portanto, esses parâmetros podem ter sido fundamentais para a ocorrência desses fungos nas áreas onde houveram o maior número de indivíduos.

Os fungos ocorrem em substratos específicos, sendo estes, determinantes na diversidade das espécies. A riqueza e a abundância das espécies de fungos estão diretamente relacionadas com a disponibilidade desses substratos (Honório \& Pasin, 2016). A serrapilheira foi o substrato que apresentou a maior riqueza de gêneros (11 gêneros totalizando 19 indivíduos), e os tocos a maior abundância (cinco gêneros e uma espécie totalizando 51 indivíduos). 
Tabela 4. Condições climáticas (temperatura do $\operatorname{ar}\left({ }^{\circ} \mathrm{C}\right)$ e umidade relativa do $\operatorname{ar}($ UR $(\%)$ ) de três áreas de reflorestamento de Eucalyptus spp. obtidas em diferentes coletas de basidiomicetos macroscópicos.

\begin{tabular}{ccccccccc}
\hline \multirow{2}{*}{ Coletas } & \multicolumn{3}{c}{ Temperatura $\left({ }^{\circ} \mathbf{C}\right)$} & & \multicolumn{3}{c}{ UR (\%) } \\
\cline { 2 - 3 } \cline { 7 - 8 } & Área A & Área B & Área C & & Área A & Área B & Área C \\
\hline 1 & $*$ & 21,3 & 26 & & $*$ & 35 & 33 \\
2 & $*$ & 27,3 & 22 & & $*$ & 36 & 38 \\
3 & $*$ & 26,0 & 25 & & $*$ & 60 & 55 \\
4 & $*$ & 28 & 22 & & $*$ & 59 & 38 \\
5 & 33 & 33,8 & 31 & & 56 & 64 & 70 \\
6 & 28 & 27,6 & 28 & & 68 & 74 & 74 \\
7 & 36 & 33,9 & 33 & & 44 & 57 & 56 \\
8 & 34 & 29,1 & 31 & & 61 & 69 & 67 \\
9 & 30 & 27,8 & 28 & & 58 & 64 & 59 \\
10 & 34 & 29,1 & 31 & & 45 & 50 & 46 \\
11 & 31 & 31,8 & 31 & & 56 & 55 & 59 \\
12 & 22 & 21,6 & 22 & & 64 & 62 & 65 \\
13 & 23 & 18,7 & 20 & & 74 & 73 & 73 \\
14 & 34 & 31 & 30 & & 45 & 46 & 45 \\
15 & 26 & 26,8 & 28 & 56 & 47 & 49 \\
16 & 22 & 22,7 & 23 & 64 & 70 & 67 \\
\hline
\end{tabular}

Nota: $\mathrm{A}$ área $\mathrm{A}$ se refere a área dos tocos pós-colheita, a área $\mathrm{B}$ a área dos tocos com um ano após a colheita e a área $C$ a área dos tocos com dois anos após a colheita. $\left.{ }^{*}\right)$ Dados não coletados.

Por meio do modelo de regressão linear simples foi possível notar que o número de indivíduos aumentou com o passar do tempo (dias após a colheita de eucalipto). A variável densidade acumulada (número de indivíduos) de fungos basidiomicetos macroscópicos está diretamente relacionada com o passar do tempo (dias após a colheita de eucalipto), ou seja, conforme aumentam os dias após a colheita, aumenta a ocorrência dos fungos (Figura 2).

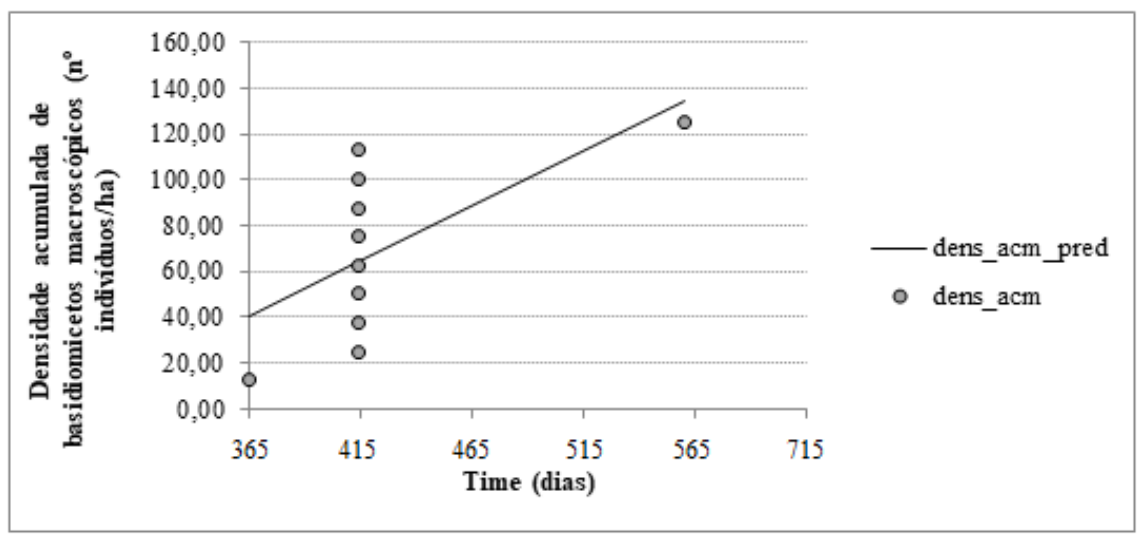

Figura 2. Modelo de regressão linear simples adotado na área 2 (tocos com um ano após a colheita) para a densidade acumulada de indivíduos no tempo (dias).

Por meio do modelo de regressão não linear logístico foi possível observar que há uma tendência de estabilização da densidade acumulada dos fungos basidiomicetos macroscópicos em relação à ocorrência desses fungos nas áreas de reflorestamento de eucalipto em torno de 836 dias após a colheita do eucalipto (Figura 3). 


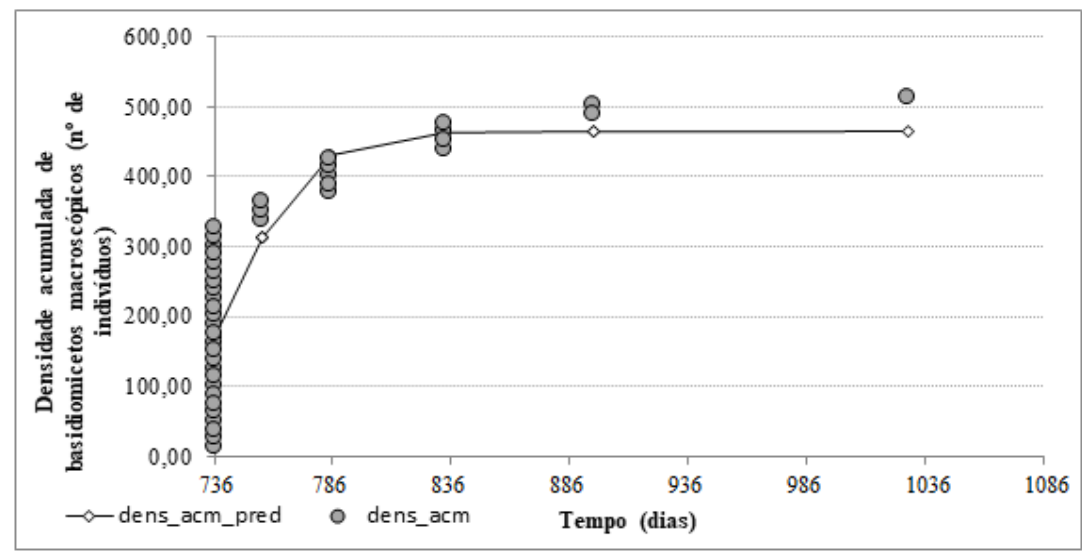

Figura 3. Modelo de regressão não linear logístico adotado na área 3 (tocos com dois anos após a colheita) para a densidade acumulada de indivíduos no tempo (dias após a colheita de eucalipto).

\section{CONCLUSÃO}

A idade dos tocos de eucalipto influenciou na ocorrência de fungos basidiomicetos macroscópicos em áreas de reflorestamento.

Os fungos do gênero Coprinus ocorreram apenas nas áreas com tocos com um ano após a colheita e os fungos Fuscoporia spp. e Pycnoporus sanguineus apenas na área com os tocos com dois anos após a colheita.

Na serrapilheira da área com os tocos com um ano após a colheita, ocorreram apenas os fungos Gloeoporus spp., Microporellus spp. e Phelinus spp. e os fungos Lentinus spp., Scleroderma spp. e Trametes spp. apenas na serrapilheira da área com os tocos com dois anos após a colheita. O único fungo encontrado em ambas as áreas foi Polyporus spp.

Os fungos mais encontrados nas áreas de reflorestamento de eucalipto durante as coletas realizadas foram Coprinus spp. e Ganoderma spp.

\section{REFERÊNCIAS}

Abrahão, M. C., Gugliotta, A. M., \& Bononi, V. L. R. (2012). Xylophilous Agaricomycetes (Basidiomycota) of the Brazilian Cerrado. Check List, 8(6), 1102-1116. http://dx.doi.org/10.15560/8.6.1102.

Alonso, S. K., Silva, A. G., Kasuya, M. C. M., Barros, N. F., Cavallazzi, J. R. P., Bettucci, L., Lupo, S., \& Alfenas, A. C. (2007). Isolamento e seleção de fungos causadores de podridão branca da madeira em florestas de Eucalyptus sp. com potencial de degradação de cepas e raízes. Revista Árvore, 31(1), 145-155. http://dx.doi.org/10.1590/S0100-67622007000100016.

Blanchette, R. A. (2000). A review of microbial deterioration found in archaeological wood from different environments. International Biodeterioration \& Biodegradation, London, 46, 189-204.

Casselli, V., Prata, G. A., \& Seixas, F. (2018). Rendimento operacional e viabilidade econômica na colheita de tocos de eucalipto em dois sistemas extração. Scientia Forestalis, 117(46), 97-106. http://dx.doi.org/10.18671/scifor.v46n117.09.

Costa, A. C. F., Marino, R., Silva, G. A., Almeida, T. A., Nascimento, K. S., \& Mesquita, J. B. (2011). Ocorrência de fungos macroscópicos em povoamentos de Eucalipto. Natural Resources, 1(1).

Empresa Brasileira de Pesquisa Agropecuária - EMBRAPA. (2014). Cultivo de eucalipto em propriedades rurais: diversificação da produção e renda. Transferência de Tecnologia Florestal. Brasília, DF: EMBRAPA.

Foelkel, C. (2014). O problema dos tocos residuais das florestas plantadas de eucaliptos. Eucalyptus Newsletter, 45, 1-17.

Furtado, J. S. (1981). Taxonomy of Amauroderma (Basidiomycetes, Polyporaceae). Memoirs of the New York Botanical Garden, 34, 1-109.

Gibertoni, T. B., Ryvarden, L., \& Cavalcanti, M. A. Q. (2004). New records of Aphyllophorales (Basidiomycota) in the Atlantic Rain Forest in Northeast Brazil. Acta Botanica Brasílica, 18(4), 975979. http://dx.doi.org/10.1590/S0102-33062004000400027. 
Honório, M. C., \& Pasin, L. A. A. P. (2016). Diversidade de fungos macroscópicos na floresta ombrófila mista do Pico da Bandeira em Maria da Fé. In Anais do XIII Congresso Nacional de Meio Ambiente de Poços de Caldas. Poços de Caldas.

Indústria Brasileira de Árvores - IBÁ. (2017). Relatório IBÁ 2017. Brasília, DF.

Madigan, M. T., Martinko, J. M., Bender, K. S., Buckley, D. H., Stahl, D. A., \& Brock, T. (2016). Microbiologia de Brock (14. ed.). Porto Alegre: ArtMed.

Magalhães, P. O., \& Milagres, A. M. F. (2008). Importância das celulases produzidas por basidiomicetos causadores de podridão branca na biodegradação de lignocelulósicos. Microbiologia em Foco, 4, 4-12.

Mora, A. L., \& Garcia, C. H. (2000). A cultura do Eucalipto no Brasil. São Paulo: Sociedade Brasileira de Silvicultura.

Moreschi, J. C. (2013). Biodegradação e preservação da madeira (v. 1, 4 ed.). Paraná: Departamento de Engenharia e Tecnologia Florestal da UFPR.

Motato-Vásquez, V., Pires, R. M., \& Gugliotta, A. M. (2014). Polypores from an Atlantic rainforest area in southeast Brazil: pileate species. Revista Brasileira de Botânica, 38(1), 149-164. http://dx.doi.org/10.1007/s40415-014-0109-7.

Negrão, D. R., Silva Júnior, T. A. F., Passos, J. R. S., Sansígolo, C. A., Minhoni, M. T. A., \& Furtado, E. L. (2014). Biodegradation of eucalyptus urograndis wood by fungi. International Biodeterioration \& Biodegradation, 89, 95-112. http://dx.doi.org/10.1016/j.ibiod.2014.01.004.

Ryvarden, L. (2004). Neotropical polypores: part 1 (Synopsis Fungorum, No. 19). Germany: NHBS.

Seber, G. A. F., \& Wild, C. J. (2003). Nonlinear regression. New York: John Wiley \& Sons.

Wilcken, C. F., Lima, A. C. V., Dias, T. K. R., Masson, M. V., Ferreira Filho, P. J., \& Pogetto, M. H. F. A. D. (2008). Guia prático de manejo de plantações de Eucalipto. Botucatu: Fundação de Estudos e Pesquisas Agrícolas e Florestais.

Contribuição dos autores: BLM: investigação, escrita - primeira redação, escrita - revisão e edição, CLM: investigação, escrita - revisão e edição, PLS: metodologia, JRSP: curadoria de dados, TAFSJ: metodologia, administração do projeto, supervisão. 